\title{
Third-order double-achromat bunch compressors for broadband beams
}

\author{
Jonas Björklund Svensson $\odot,{ }^{1, *}$ Tessa K. Charles, ${ }^{2,3}$ Olle Lundh $\odot,{ }^{1}$ and Sara Thorin ${ }^{4, \dagger}$ \\ ${ }^{1}$ Department of Physics, Lund University, P.O. Box 118, SE-22100, Lund, Sweden \\ ${ }^{2}$ CERN, 1211 Geneva 23, Switzerland \\ ${ }^{3}$ School of Physics, University of Melbourne, 3010, Victoria, Australia \\ ${ }^{4}$ MAX IV Laboratory, Lund University, P.O. Box 118, SE-22100, Lund, Sweden
}

(Received 12 July 2019; published 23 October 2019)

\begin{abstract}
Many state-of-the-art applications for linear accelerators, such as free-electron lasers (FELs) and plasmawakefield accelerators (PWFAs), require small normalized emittances, and PWFAs in particular are very sensitive to transverse slice offsets along the beam. Dispersive systems, such as bunch compressors, can cause different chromatic aberrations, one of which yields transverse slice offsets. In this paper, we show a design approach to double-achromat bunch compressors which greatly reduces different chromatic aberrations and mitigates coherent synchrotron radiation effects.
\end{abstract}

DOI: 10.1103/PhysRevAccelBeams.22.104401

Bunch compressors (BCs) are an essential part of many linear electron accelerators and are vital for most state-ofthe-art applications, such as free-electron lasers (FELs), linear colliders (LCs) and beam-driven plasma-wakefield accelerators (PWFAs). As a correlated energy spread, or chirp, along the beam is often required to compress particle bunches to the desired duration or peak current, compressors and transport optics exhibiting chromatic aberrations can cause different beam parameters to change along the beam. Particularly beams entering and exiting PWFAs can exhibit large energy spreads, which exacerbates the chromatic effects.

Similarly to chromatic aberrations in photon optics, different longitudinal slices of a chirped particle beam will be focused at different longitudinal positions for given focusing optic. This causes the beam Twiss parameters to change along the beam, which can cause poor matching conditions and focusing, as well as a growth of the projected normalized emittance. This type of aberration is generally called chromaticity, and a system which mitigates this aberration using only linear focusing elements is called apochromatic. In systems containing dipole magnets, such as bunch compressors and transfer beamlines, another type of aberration can cause different slices to also exit the system with a different centroid, or mean, position and angle. Beyond also causing projected normalized emittance

\footnotetext{
*jonas.bjorklund_svensson@fysik.lth.se

sara.thorin@maxiv.lu.se
}

Published by the American Physical Society under the terms of the Creative Commons Attribution 4.0 International license. Further distribution of this work must maintain attribution to the author(s) and the published article's title, journal citation, and DOI. growth, this type of transverse offset from the axis has been shown to decrease FEL performance [1], while for PWFAs [2,3], it can cause beam hose instability [4], which can completely destroy the beam. This aberration can be caused by nonzero, or leaking, transverse dispersion, and a system which is devoid of such dispersion to order $n$ is typically called $n$ th-order achromatic.

Much work has been devoted in the past few years to understanding and mitigating the effects of the hose instability $[5,6]$, but if the transverse offset can be removed before entering the plasma, the root cause of the instability is also removed. Leaking higher-order dispersion has been identified as a potential issue for staging in future PWFAbased LCs [7]. Large energy spread in the beam also, in itself, serves to mitigate hosing growth, as shown in Ref. [5]. Therein it is also shown that controlled plasma density tapers, which are also useful for matching the beam parameters from vacuum to plasma [8,9], are useful for mitigating hosing, and are likely to be implemented in future PWFA experiments.

In this paper, we show by means of numerical simulations in Elegant [10], a design approach to doubleachromat bunch compressors [11] which are achromatic to third order, while also exhibiting apochromatic properties. We show that these systems can transport and compress beams with significant energy spreads and can be tuned to mitigate some effects of coherent synchrotron radiation (CSR), which is always present in such systems and can cause spatial and angular offsets similar to leaking higher-order dispersion. Double-achromat bunch compressors have the added benefit that they can easily be optically tuned to provide linearly ramped beam current profiles [12], which are nearly optimal for efficiently driving plasma waves [13-15], requiring no harmonic cavity to do so. These features allow double-achromats to be 


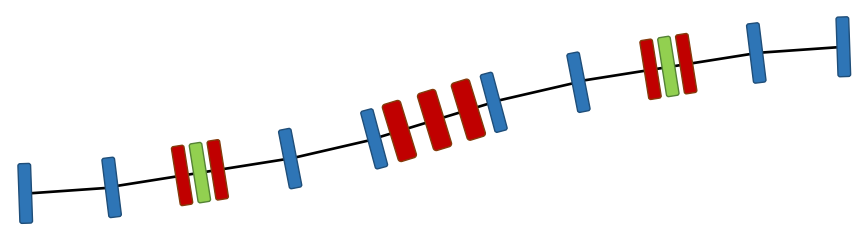

FIG. 1. Overview of the geometry of a double-achromat compressor. Blue rectangles are dipoles, red are quadrupoles and green are sextupoles. This color convention will be the same for all following figures.

configured as primary bunch compressors or truly transparent (with respect to energy spread) transfer beamlines, with or without current profile shaping. These facts present a strong case for this bunch compressor scheme in, e.g., future PWFA machines. An example of the geometry of a double-achromat compressor (MAX IV BC1) is shown in Fig. 1.

In the following, the matrix-based beam transfer notation of Brown and Carey $[16,17]$ will be used. In this notation, the transformation $X \rightarrow X_{f}$ of the $6 \mathrm{D}$ particle coordinate vector $X=\left(x, x^{\prime}, y, y^{\prime}, z, \delta\right)$ between two points along the lattice is described by

$X_{i, f}=\sum_{j} R_{i j} X_{j}+\sum_{j k} T_{i j k} X_{j} X_{k}+\sum_{j k l} U_{i j k l} X_{j} X_{k} X_{l}$,

where $i$ is the index of the vector $X$ and $\delta=\Delta E / E$ is the relative energy spread, with $E$ being the mean energy. $R_{i j}$, $T_{i j k}$ and $U_{i j k l}$ are the first-, second- and third-order transfer matrix elements, respectively, which depend on all the components that constitute the transport system in question. As an example, consider the mapping of the coordinate $x \rightarrow x_{f}$ as a function of the energy spread $\delta$ alone, as given by

$$
x_{f}=x+R_{16} \delta+T_{166} \delta^{2}+U_{1666} \delta^{3}=x+\Delta x(\delta),
$$

with $i=1$ and $j=k=l=6$ following the notation of Eq. (1). The linear energy dependence, $R_{16}$, is the transverse dispersion, and $T_{166}$ and $U_{1666}$ are the second- and third-order transverse dispersion, respectively. At either end of an achromat, $R_{16}=R_{26} \equiv 0$, and so we call a lattice with $T_{166}=T_{266}=0$ at these locations a second-order achromat, and so on. Passing through a system with nonzero dispersion yields $\Delta x \neq 0$ for $\delta \neq 0$, which produces an offset of the slices in a chirped beam, as particles within a slice have nearly the same energy.

An example of this is shown in Fig. 2, which shows the horizontal slice centroids of a single bunch with large energy spread which is tracked through a first-order double-achromat bunch compressor, together with the predicted offset from Eq. (2). There are evidently remaining $T_{166}$ and $U_{1666}$ elements, as $\Delta x(\delta)$ takes the shape of a skewed parabola. Other effects, such as CSR and

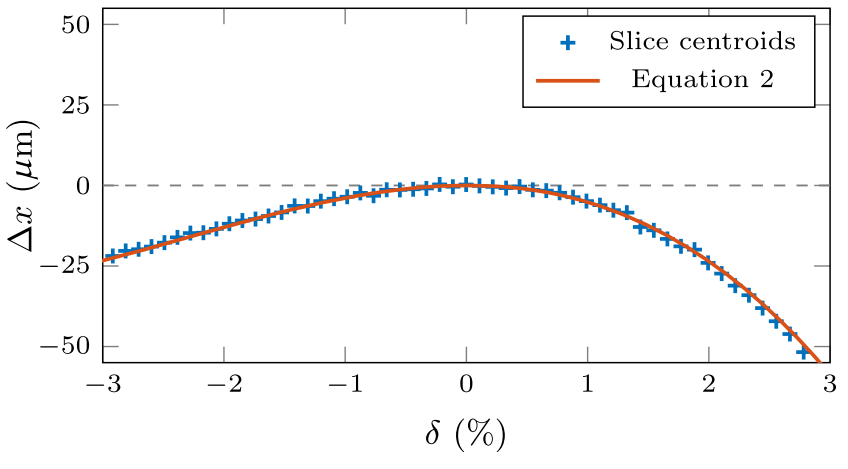

FIG. 2. Horizontal offset of the beam after the BC, as a function of $\delta$. The blue marks show the slice centroids of the tracked beam and the red line is Eq. (2) with the corresponding coefficients for the $\mathrm{BC}$ in question.

wakefields, are turned off to show only the coupling between the energy spread and the offset.

The matrix elements $T_{266}$ and $U_{2666}$, which relate energy spread and angular deviation similarly to Eq. (2), are also of interest, as are the elements $T_{566}$ and $U_{5666}$, which relate energy spread and longitudinal position in the bunch. $R_{56}$ is the first-order longitudinal dispersion, so $T_{566}$ and $U_{5666}$ are thus higher-order longitudinal dispersion, which can be tuned to particular values for shaping of the beam current profile. The longitudinal dispersion elements one might want to control and give some specific, nonzero value, while it is often desirable to cancel the transverse elements mentioned above.

All these different matrix elements are determined by the magnets in the setup, but different magnets affect different order elements. Assuming that the magnets are perfectly aligned, dipoles affect terms from 0 (trajectory deflection) and up, quadrupoles from 1 and up, sextupoles from 2 and up, etc. Misalignment will cause a feed-down effect [18], which means that a magnet of given order will also exhibit lower-order terms; a transversely displaced quadrupole will also give a dipole kick. This effect will be disregarded in this paper for simplicity. Finite manufacturing tolerances also introduce different-order terms, also known as the multipole content [18] of the magnet. The specific multipole content for the MAX IV compressor magnets is included in the numerical tracking throughout this paper. Regardless, a magnet of a given order can typically be used to tune a matrix element of the same order, but it will also affect all higher order as its strength and longitudinal position is changed. It is generally a good idea to optimize the setup using lower-order magnets first.

We also note that the cross-terms, such as $T_{116}$, which maps to the transverse coordinate by $\Delta x=T_{116} x \delta$, are omitted in the analytical work. While in principle contributing to emittance increase, the initial position in, e.g., $x$ is small enough in the cases considered here that the resulting $\Delta x$ is very small compared to the beam size and secondly, 
terms such as this give a symmetric shift for particles at positive and negative $x$, and will therefore not give a collective centroid offset. This is also true for similar terms related to the angular offset, $\Delta x^{\prime}$.

The starting point of the design is the second bunch compressor in the MAX IV Laboratory [19] linear accelerator [20], see Fig. 3. The compressors are self-linearizing with respect to the longitudinal $(z-\delta)$ phase space, and so no harmonic linearizing cavity, commonly used in chicanebased linacs, is required. Unlike the more common chicanes, this type of bunch compressor has a positive $R_{56}$, which means that a positive chirp with respect to $z$ is required for compression. The compressors self-linearize the longitudinal phase space of the bunches by tuning $T_{566}$ with the weak $\left(\left|k_{2}\right| \leq \sim 70 \mathrm{~m}^{-3}\right)$ sextupoles at the center of each achromat. These sextupoles can also be used to easily adjust the bunch current profile [12]. $R_{56}$ is fixed at a value of $2.60 \mathrm{~cm}$ for the full compressor and $R_{16}=R_{26}=0$ at each achromat end. The degree of compression is varied with the energy chirp, which is established with the phase of the accelerating radio frequency (rf) voltage as well as

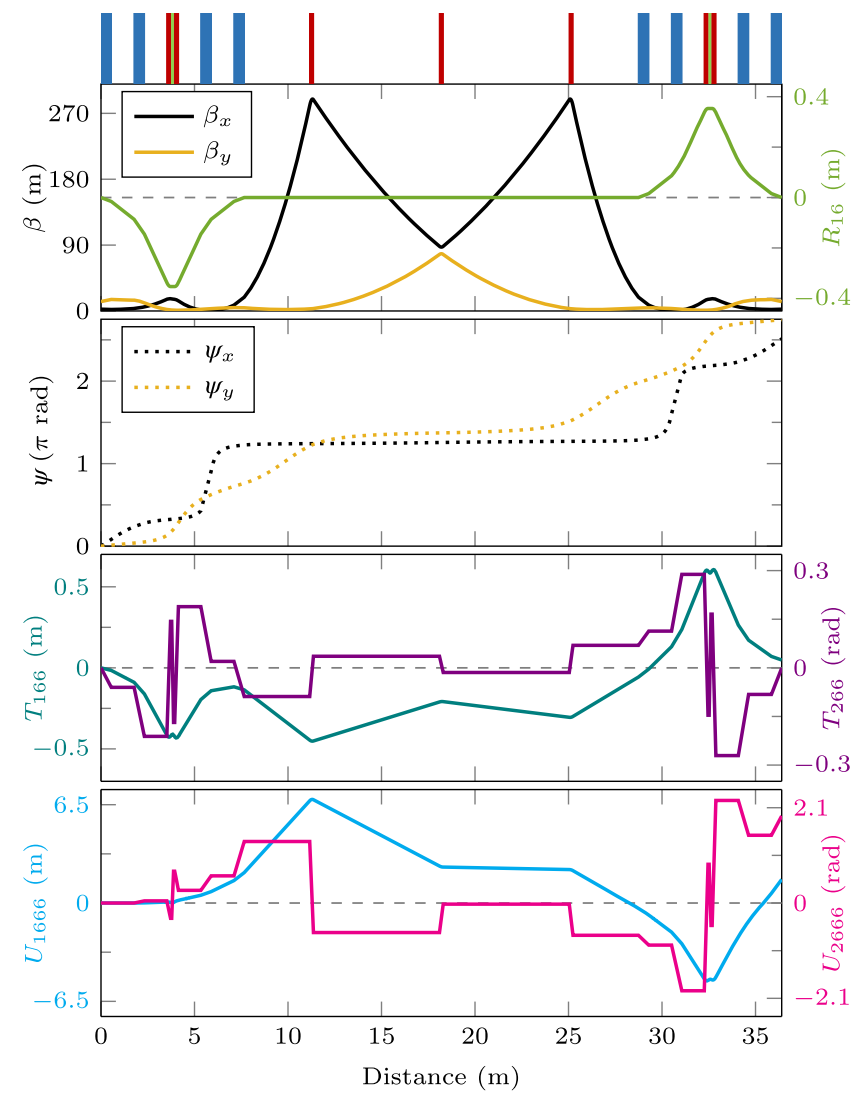

FIG. 3. Original BC2 layout (top) followed by beta functions $\left(\beta_{x}\right.$ and $\left.\beta_{y}\right)$ and dispersion $\left(R_{16}\right)$, betatron phase advances $\left(\psi_{x}\right.$ and $\left.\psi_{y}\right)$, second-order spatial and angular dispersion $\left(T_{166}\right.$ and $\left.T_{266}\right)$, and finally third-order spatial and angular dispersion $\left(U_{1666}\right.$ and $U_{2666}$ ). Note the asymmetric behavior of the higher-order dispersion curves. the longitudinal wakefield of the bunch, which chirps in the same direction as the rf.

Third-order achromaticity is achieved in a few key steps. First, two new quadrupoles are introduced in the straightsection between the achromats. Their strengths and relative positions are optimized, using the Simplex optimizer in Elegant, to minimize $\left|T_{166}\right|$ and $\left|T_{266}\right|$ at the compressor end, while keeping the Twiss functions symmetric about the compressor center. This reduces not only $\left|T_{166}\right|$ and $\left|T_{266}\right|$, but also $\left|U_{1666}\right|$ and $\left|U_{2666}\right|$, decreasing $\left|U_{5666}\right|$ only very slightly. $T_{566}$ is unaffected. A benefit of using the straight-section quadrupoles for this type of task is that it has negligible effect on the overall compression, even to third order. Then, the sextupole configuration is changed to have sextupoles from two different families placed symmetrically around the achromat centers, outside the achromat quadrupoles that control the first-order dispersion, while an octupole magnet is placed in the center, see Fig. 4 top. The sextupoles and octupoles in the second achromat are configured to have the opposite sign of the strength compared to the first achromat. Starting the tuning with all

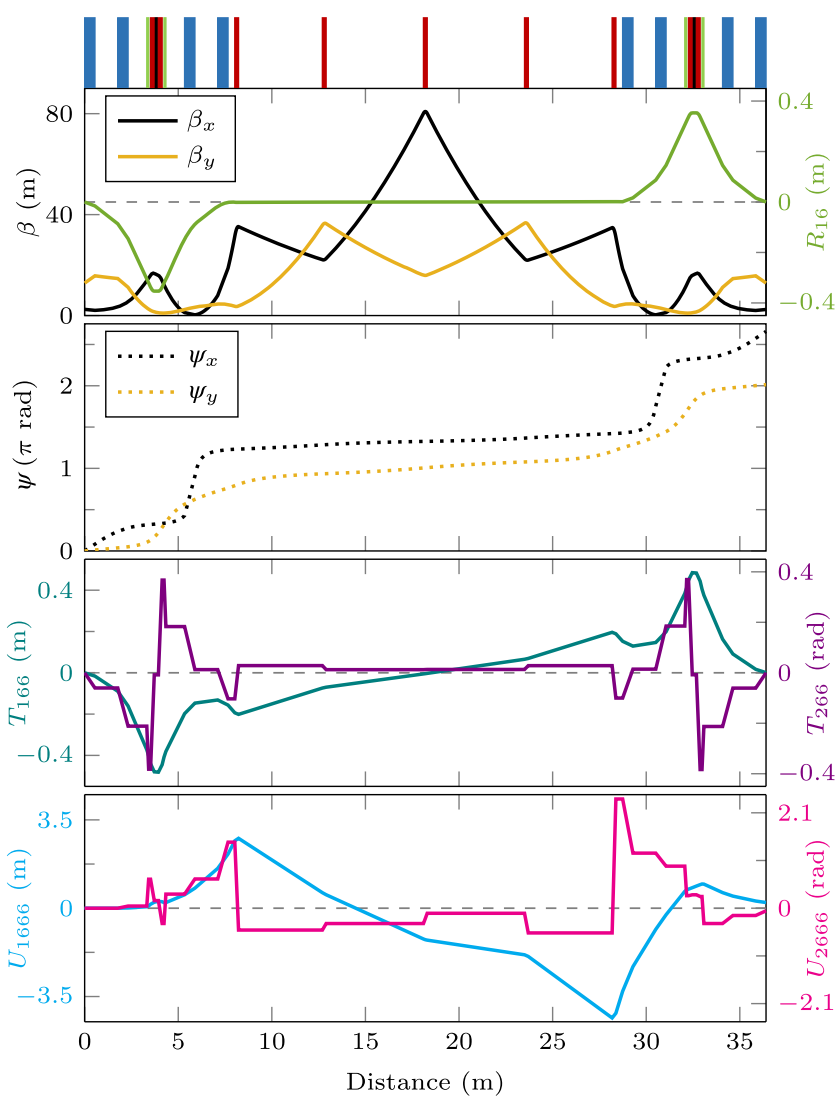

FIG. 4. CSR-mitigating and near-apochromatic BC. Again with compressor layout on top, followed by beta functions and dispersion, betatron phase advances, second-order spatial and angular dispersion, and finally third-order spatial and angular dispersion. The added octupoles are shown in black. Note the $\Delta \psi_{x}=2 \pi$ phase advance between achromat centers. 
higher-order magnets set to 0 , the two sextupole families are used to set $T_{566}$ to its nominal value of $4.23 \mathrm{~cm}$, after which $\left|T_{166}\right|$ and $\left|T_{266}\right|$ are again minimized using the 5 straight-section quadrupoles. Then, the octupoles are used to minimize $\left|U_{1666}\right|$ and $\left|U_{2666}\right|$, after which all magnets and the straight-section drifts are simultaneously used to set these four matrix elements to zero.

The resulting spatial offset of the slices out of the compressor [using Eq. (2)] is then $\ll 1 \mu \mathrm{m}$, and the angular offset was $\ll 1 \mu \mathrm{rad}$, at $\delta= \pm 3 \%$. The higher-order magnets are very weak, the sextupole strengths being 30 and $42 \mathrm{~m}^{-3}$, respectively, and the octupole strength being $0.7 \mathrm{~m}^{-4}$. Removing the octupole altogether does not allow for convergence of the $U$-terms, possibly because of the dipole limitation mentioned earlier. The compressor footprint is unchanged. The offset in a beam tracked through the compressor is shown and compared to Eq. (2) in Fig. 5. The small discrepancy is a result of third-order $(U)$ matrices for the dipole magnets not being implemented in Elegant. The two sextupole families, together with the octupole and the three families of quadrupoles, allow for tuning $T_{566}$ to account for different voltage curvature at different $\mathrm{rf}$ phases, while minimizing the remaining $T$ - and $U$-terms. Since the sextupoles are also used to, e.g., overlinearize the longitudinal phase space, to yield the linear current ramps shown in, e.g., Ref. [12], this approach could be

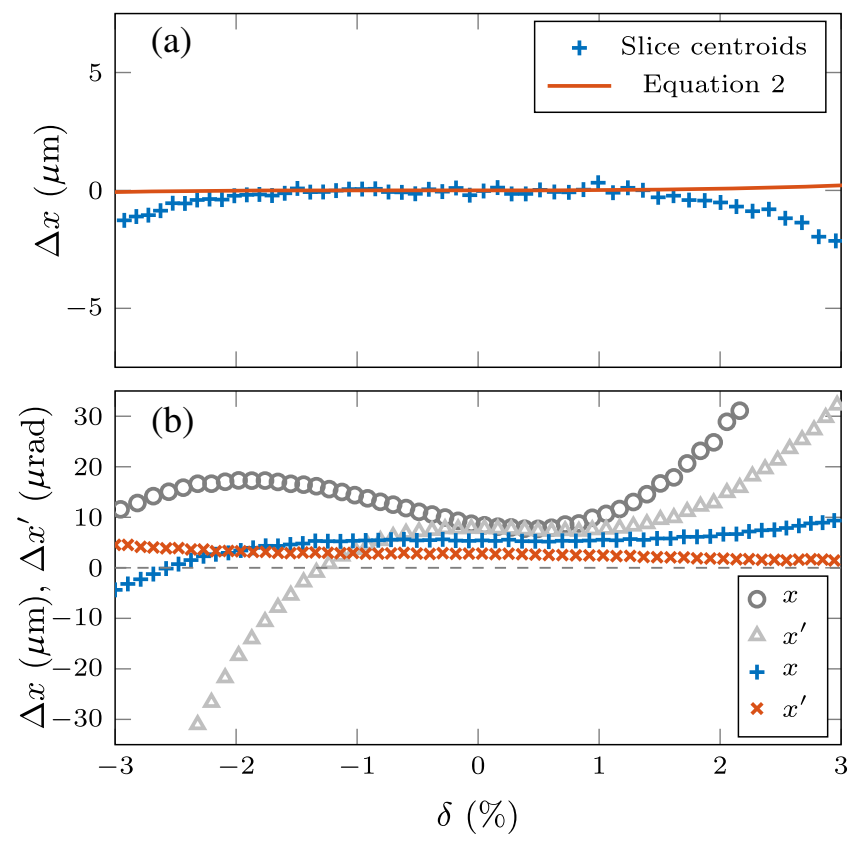

FIG. 5. (a) Horizontal offset of the beam after the third-order achromatic BC as a function of $\delta$. The blue marks show the slice spatial centroids of a tracked beam and the red line is Eq. (2), using the corresponding matrix elements. Note the large difference in scale compared to Fig. 2. (b) Horizontal slice spatial and angular centroids after tracking through the lattices in Fig. 3 (gray circles and triangles) and Fig. 4 (colored crosses), with CSR enabled. used to construct, e.g., PWFA-tailored, offsetfree bunch compressors.

An alternative design approach could be the one taken in Ref. [21], particularly considering the very similar dogleg case in Sec. V of that paper. As noted, there is a positive $R_{56}$ of such a double-achromat, which could possibly be increased from the presented $5 \mathrm{~mm}$ to some desirable value $[O(\mathrm{~cm})]$ and still keep the achromats in, or near, the regime for second-order achromaticity. Leaving out the added chicane, one ends up precisely with a doubleachromat compressor. It is also noted in the paper that there is some residual $T_{566}$, which could be tuneable by weak sextupoles in a similar manner to the MAX IV compressors, if it cannot indeed be increased by the linear magnet layout alone. Two sextupole families would be required, then, to set $T_{566}$ and minimize $T_{166}$ or $T_{266}$. The design presented in Ref. [21] is also scalable in length via the center straight-section, just like the layouts presented here, which could then also provide additional knobs for minimizing $U_{1666}$ and $U_{2666}$ (since $T_{166}$ and $T_{266}$ are already zero), similarly to what was described above.

Up until now, we have considered the effects of the higher-order transverse dispersion arising in compressors. However, there are of course also chromatic aberrations in nondispersive systems. These effects are often compared to the chromatic aberrations arising in photon optics, where different photon energies, or wavelengths, usually have different focal lengths for a given lens. Normally, these aberrations in charged particle optics can be canceled by the use of sextupoles in dispersive sections (see e.g., Refs. $[22,23])$, but it is also possible to mitigate them by the use of linear focusing elements, such as quadrupoles, alone.

Using the definition of Ref. [24], an optical layout where the first-order chromatic derivatives of the Twiss functions, i.e., $\frac{d \beta}{d \delta}$ and $\frac{d \alpha}{d \delta}$, are cancelled by linear focusing elements, is called first-order apochromatic. This approach is very useful for transport beamline and final focus design, as the Twiss functions, between two given locations along the system, can be conserved, in spite of a significant energy spread. If the magnet layout is chromatic, a beam with a correlated energy spread will acquire different Twiss parameters along the beam, which can lead to poor transport and focusing, which then leads to, e.g., projected emittance growth. It is, of course, particularly important to take this into account in sections of the system where the energy spread is the large, just as for the higher-order dispersion.

Incidentally, the center straight-section quadrupoles are useful not only for controlling the higher-order dispersion, but also for reducing the overall chromaticity. This means that if one has the headroom in terms of degrees-of-freedom, these magnets can be used for multiple purposes. E.g., if one uses the design principle of Ref. [21], and third-order terms are not a concern, the 
center straight-section is free to use for canceling the chromatic derivatives. Alternatively, one can opt for controlling the betatron phase advance between achromats with these quadrupoles-this can be an implicit effect when cancelling the chromatic derivatives, as shown in Ref. [24]. It is worth noting that it can be worth limiting the maximum beta function value along the system, as this was found to flatten the beta responses as function of $\delta$, particularly in the nonbending plane. Even with the Twiss derivatives zeroed at $\delta=0$, rapid increase of the Twiss functions for $\delta \neq 0$ would yield chromatic aberrations in the beam.

So far, only the optical functions have been considered, and unwanted chromatic effects in the beam have been minimized. However, there are other effects, not contained within the previously used theory, that can have a detrimental impact on the final beam quality. One such effect is coherent synchrotron radiation (CSR). This electromagnetic radiation is generated (mostly) in the dipole magnets, where the beam is deflected by a large amount, and is coherent for wavelengths that are on the order of twice the bunch length, or features in the bunch current, and longer. The CSR can both increase the slice energy spread and emittance on the bunch, and also impart a transverse momentum, or kick, along it. This can lead to similar offsets in position and angle to was described earlier, so clearly, these are also effects that need to be limited.

To control these effects, we note that the CSR will impart a spatial and angular offset on the beam at the end of the compressor, which can be approximated by third-order polynomials. Because of this, one can combat this effect by detuning the dispersion functions using quadrupoles, sextupoles and octupoles, to mitigate linear, quadratic and cubic terms, respectively; here the dispersion quadrupoles in the achromats are detuned to this end (which does affect $R_{56}$ slightly), but dipole corrector magnets might be more appropriate as they could also help remove constant terms. Using the straight-section quads is effective because of how they transport the higher-order dispersion functions, but they also affect the betatron phase advance between the achromats, which is a known strategy for mitigating CSR kicks. The apochromaticity, compared to the original and third-order lattices, is also improved by using these quadrupoles (cf. Fig. 6). The final lattice is shown in Fig. 4. Note that the second-order dispersion is almost symmetric, while the third-order dispersion was detuned to a greater extent. For the third-order achromatic compressor, all second- and third-order functions are (anti-)symmetric around the compressor center.

As a final benchmark, we simulate bunch compression using this design with a $3 \mathrm{GeV}$ beam of $250 \mathrm{pC}$ charge, $0.5 \mathrm{~mm}$ mrad normalized emittance and with a Gaussian current profile, from $3.15 \mathrm{ps}$ fwhm down to $81.5 \mathrm{fs}$ fwhm, for a peak current of $2.66 \mathrm{kA}$. These parameters are in the

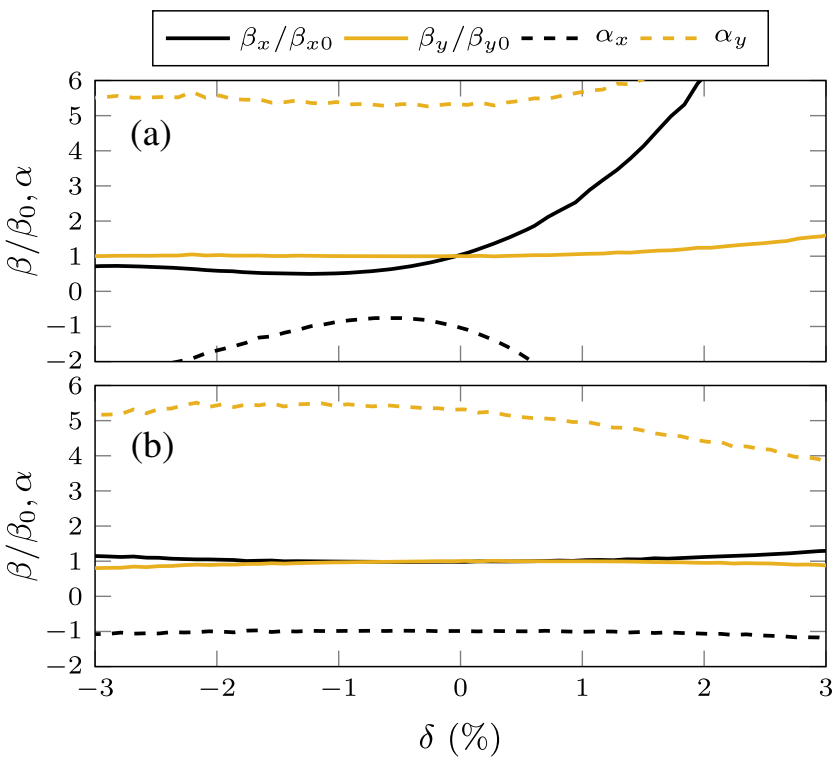

FIG. 6. Plot of the tracked slice Twiss functions as functions of $\delta$ at the end of (a) original BC2 (see Fig. 3), (b) the CSRmitigating modification of the third-order compressor (see Fig. 4).

realm of PWFA drivers, a scheme which is more sensitive to the transverse offset than, e.g., FEL, in that it can be a "make-or-break"-type scenario. The longitudinal phase space curvature at the entrance of the compressor corresponds to accelerating at 30.5 degrees off-crest, yielding an energy spread of $1.61 \% \mathrm{rms}$, or $6.6 \%$ end-to-end. $T_{566}$ is changed from its nominal value of $4.23 \mathrm{~cm}$ to $3.58 \mathrm{~cm}$, using the sextupoles, to accommodate the different rf curvature and linearize the final longitudinal phase space; the remaining magnets are re-tweaked once the desired rf phase and $T_{566}$ are known so that the higher-order transverse dispersion is well controlled and the transverse emittance in minimized. The sextupoles and octupoles are still weak, at 37.6 and $34.8 \mathrm{~m}^{-3}$ and $0 \mathrm{~m}^{-4}$, respectively. $10^{6}$ macroparticles were used for the tracking simulations.

The resulting offset after compression is shown in Fig. 5(b), with the tracked slice Twiss parameters displayed as a function of $\delta$ in Fig. 6(b). Evidently, $\Delta x$ and $\Delta x^{\prime}$ are not altogether completely removed, but they are much flatter than before. Constant offsets like these could be improved using dipole corrector magnets. When undergoing transport, such as focusing into a plasma cell, these parameters will change somewhat, but the ratio of $\Delta x$ to the transverse beam size $\sigma_{x}$ will remain constant, as they are (de-) magnified by the same factor. As an example, in Ref. [6] it is shown that for an initial transverse offset of $0.2 \sigma_{x}$, the oscillations are damped and the instability does not grow. Examining the fraction of our bunch within $\delta= \pm 3 \%$ (93\% of the total charge), $\sigma_{x}=14.8 \mu \mathrm{m}$, the weighted (by current) mean absolute offset is $5.27 \mu \mathrm{m}$ and the weighted deviation from this mean is $1.21 \mu \mathrm{m} \mathrm{rms}$, so 
that this deviation is $<0.1 \sigma_{x}$. Only at the tail of the current distribution $(\delta<0)$ is the deviation from the mean larger, for a small fraction of the total charge. The horizontal normalized projected emittance $\epsilon_{n x}$ grows by $3.6 \%$.

Worth noticing here is that while these final results were obtained numerically, the results are well understood from analytic theory. A rigorous analytical analysis, such as the one presented in Ref. [25], could yield further insight and potential developments still. As is well known from matrix treatments of beam transport (see e.g., Ref. [26]), a $-I$ transformation (i.e., a betatron phase shift of $\pi$ ) between two identical sextupoles serves to cancel nonlinear kicks imposed by the first sextupole, mitigating also geometric aberrations. In our case, the optimal phase shift is instead $2 \pi$, corresponding to an $I$-transformation, as the sextupoles (and octupoles) in the two achromats are of equal magnitude but opposite sign. This is indeed the achieved phase advance in $x$ between the achromat centers in the new version (Fig. 4), as opposed to $1.87 \pi$ previously (Fig. 3). While $\Delta \psi_{y}$ is not a multiple of $\pi$ between the achromat centers, $\beta_{y}$ is small enough at the location of all higherorder magnets that the $\psi_{y}$-dependent terms only contribute by a small amount, even to fourth order as suggested by Eq. (3.16) in Ref. [25]. Since the final layout is optimized partially for tracked beam emittance, the lack of dipole $U$-terms does not interfere in the same way as before. The octupole is still useful for flexibility in different use-cases, e.g., tuning the third-order component of the slice offset, and is therefore not removed. The end result is that projected emittance growth induced by geometric aberrations from nonlinear magnets is also reduced significantly, allowing for control of the higher-order transverse and longitudinal dispersion terms without sacrificing the beam emittance.

In summary, we have shown a double-achromat design which is achromatic to third order and which has apochromatic properties, as well as the optimization approach to reach it. The compressor design can also be used to mitigate CSR effects, which will always be present in real systems, as well as geometric aberrations from the higher-order magnets. The applications of such bunch compressors or similar beam transport systems are many, but a key one is for PWFA experiments, because of the ease of current shaping and the possibility to minimize the transverse offset and other chromatic effects, for which PWFA is generally rather sensitive.

\section{ACKNOWLEDGMENTS}

The authors thank Carl Andreas Lindstrøm for helpful discussions about apochromatic lattices. We also thank Michael Borland for extensive help and feedback regarding Elegant. Finally, we acknowledge the support of the Swedish Research Council and the Knut and Alice Wallenberg Foundation.
[1] M. W. Guetg, B. Beutner, E. Prat, and S. Reiche, Optimization of free electron laser performance by dispersionbased beam-tilt correction, Phys. Rev. Accel. Beams 18, 030701 (2015).

[2] P. Chen, J. M. Dawson, R. W. Huff, and T. Katsouleas, Acceleration of Electrons by the Interaction of a Bunched Electron Beam with a Plasma, Phys. Rev. Lett. 54, 693 (1985).

[3] R. Ruth, A. Chao, P. Morton, and P. Wilson, A plasma wake field accelerator, Part. Accel. 17, 171 (1985).

[4] D. H. Whittum, W. M. Sharp, S. S. Yu, M. Lampe, and G. Joyce, Electron-Hose Instability in the Ion-Focused Regime, Phys. Rev. Lett. 67, 991 (1991).

[5] T. J. Mehrling, R. A. Fonseca, A. Martinez de la Ossa, and J. Vieira, Mitigation of the Hose Instability in Plasma-Wakefield Accelerators, Phys. Rev. Lett. 118, 174801 (2017).

[6] A. Martinez de la Ossa, T. J. Mehrling, and J. Osterhoff, Intrinsic Stabilization of the Drive Beam in PlasmaWakefield Accelerators, Phys. Rev. Lett. 121, 064803 (2018).

[7] C. A. Lindstrøm, E. Adli, J. M. Allen, J. P. Delahaye, M. J. Hogan, C. Joshi, P. Muggli, T. O. Raubenheimer, and V. Yakimenko, Staging optics considerations for a plasma wakefield acceleration linear collider, Nucl. Instrum. Methods Phys. Res., Sect. A 829, 224 (2016).

[8] K. Floettmann, Adiabatic matching section for plasma accelerated beams, Phys. Rev. Accel. Beams 17, 054402 (2014).

[9] X. L. Xu, J. F. Hua, Y. P. Wu, C. J. Zhang, F. Li, Y. Wan, C.-H. Pai, W. Lu, W. An, P. Yu, M. J. Hogan, C. Joshi, and W. B. Mori, Physics of Phase Space Matching for Staging Plasma and Traditional Accelerator Components Using Longitudinally Tailored Plasma Profiles, Phys. Rev. Lett. 116, 124801 (2016).

[10] M. Borland, Elegant: A flexible SDDS-compliant code for accelerator simulation, Argonne National Laboratory Technical Report No. LS-287, 2000, https://doi.org/ 10.2172/761286.

[11] S. Thorin, M. Eriksson, S. Werin, D. Angal-Kalinin, J. McKenzie, B. Militsyn, and P. Williams, Bunch compression by linearising achromats for the MAX IV injector, in Proceedings of the 32nd Free Electron Laser Conference, Malmö, Sweden (Max-lab, Sweden, 2010), WEPB34.

[12] J. Björklund Svensson, H. Ekerfelt, O. Lundh, T. K. Charles, J. Andersson, M. Kotur, F. Lindau, E. Mansten, and S. Thorin, Beamline design for plasma-wakefield acceleration experiments at MAX IV, Proceedings of the 18th Advanced Accelerator Concepts Workshop, Breckenridge, CO, USA (IEEE, 2018), https://doi.org/10.1109/AAC.2018.8659401.

[13] K. L. F. Bane, P. Chen, and P. B. Wilson, On collinear wake field acceleration, SLAC Report No. SLAC-PUB-3662, 1985.

[14] P. Chen, J. J. Su, J. M. Dawson, K. L. F. Bane, and P. B. Wilson, Energy Transfer in the Plasma Wake-field Accelerator, Phys. Rev. Lett. 56, 1252 (1986).

[15] K. V. Lotov, Efficient operating mode of the plasma wakefield accelerator, Phys. Plasmas 12, 053105 (2005).

[16] K. L. Brown, A first- and second-order matrix theory for the design of beam transport systems and charged particle spectrometers, SLAC Report No. 75, 1982. 
[17] D. C. Carey, K. L. Brown, and F. Rothacker, Third-order TRANSPORT, a computer program for designing charged particle beam transport systems, SLAC Report No. SLACR-95-462, 1995.

[18] S. Russenschuck, Field Computation for Accelerator Magnets: Analytical and Numerical Methods for Electromagnetic Design and Optimization (Wiley-VCH, Weinheim, 2010).

[19] N. Mårtensson and M. Eriksson, The saga of MAX IV, the first multi-bend achromat synchrotron light source, Nucl. Instrum. Methods Phys. Res., Sect. A 907, 97 (2018).

[20] S. Thorin, J. Andersson, F. Curbis, M. Eriksson, O. Karlberg, D. Kumbaro, E. Mansten, D. Olsson, and S. Werin, The MAX IV linac, Proceedings of the 27th Linear Accelerator Conference, Geneva, Switzerland (2014), http://accelconf.web.cern.ch/AccelConf/LINAC2014/ papers/tuioa03.pdf.

[21] Y. Sun, Second-order achromat design based on FODO cell, Phys. Rev. ST Accel. Beams 14, 060703 (2011).
[22] P. Raimondi and A. Seryi, Novel Final Focus Design for Future Linear Colliders, Phys. Rev. Lett. 86, 3779 (2001).

[23] R. Brinkmann, P. Raimondi, and A. Seryi, Halo reduction by means of non linear optical elements in the NLC final focus system, in Proceedings of the 19th Particle Accelerator Conference, Chicago, IL, 2001 (IEEE, Piscataway, NJ, 2001), p. 3828.

[24] C. A. Lindstrøm and E. Adli, Design of general apochromatic drift-quadrupole beam lines, Phys. Rev. Accel. Beams 19, 071002 (2016).

[25] Y. Cai, K. Bane, R. Hettel, Y. Nosochkov, M.-H. Wang, and M. Borland, Ultimate storage ring based on fourthorder geometric achromats, Phys. Rev. Accel. Beams 15, 054002 (2012).

[26] R. V. Servranckx and K. L. Brown, Circular machine design techniques and tools, Nucl. Instrum. Methods Phys. Res., Sect. A 258, 525 (1987). 